\title{
Hacia un modelo predictivo de carácter preventivo del riesgo de infección por COVID-19
}

\author{
Djamel Toudert* \\ El Colegio de la Frontera Norte, Departamento de Estudios Urbanos y del Medio Ambiente, Baja California, México
}

\begin{abstract}
Resumen
Introducción: La escasez de aplicaciones centradas en la persona y con vistas al desarrollo de la conciencia del riesgo que representa la pandemia de COVID-19 estimula la exploración y creación de herramientas de carácter preventivo accesibles a la población. Objetivo: Elaboración de un modelo predictivo que permita evaluar el riesgo de letalidad ante infección por el virus SARS-CoV-2. Métodos: Exploración de datos públicos de 16000 pacientes positivos a COVID-19, para generar un modelo discriminante eficiente, valorado con una función score y que se expresa mediante un cuestionario autocalificado de interés preventivo. Resultados: Se obtuvo una función lineal útil con capacidad discriminante de 0.845 ; la validación interna con bootstrap y la externa, con $25 \%$ de los pacientes de prueba, mostraron diferencias marginales. Conclusión: El modelo predictivo, basado en 15 preguntas accesibles puede convertirse en una herramienta de prevención estructurada.
\end{abstract}

PALABRAS CLAVE: Modelo predictivo de la letalidad. COVID-19. Herramienta preventiva. México.

\section{Towards a predictive model for prevention nature of the risk of COVID-19 infection}

\section{Abstract}

Introduction: The scarcity of person-centered applications aimed at developing awareness on the risk posed by the COVID-19 pandemic, stimulates the exploration and creation of preventive tools that are accessible to the population. Objective: To develop a predictive model that allows evaluating the risk of mortality in the event of SARS-CoV-2 virus infection. Methods: Exploration of public data from 16,000 COVID-19-positive patients to generate an efficient discriminant model, evaluated with a score function and expressed by a self-rated preventive interest questionnaire. Results: $A$ useful linear function was obtained with a discriminant capacity of 0.845; internal validation with bootstrap and external validation, with $25 \%$ of tested patients showing marginal differences. Conclusion: The predictive model with statistical support, based on 15 accessible questions, can become a structured prevention tool.

KEY WORDS: Predictive model of mortality. COVID-19. Preventive tool. Mexico.

Correspondencia:

${ }^{*}$ Djamel Toudert

E-mail: toudert@ colef.mx
Fecha de recepción: 02-09-2020

Fecha de aceptación: 02-02-2021

DOI: $10.24875 /$ GMM.20000628
Gac Med Mex. 2021;157:240-245

Disponible en PubMed www.gacetamedicademexico.com

0016-3813/๑ 2021 Academia Nacional de Medicina de México, A.C. Publicado por Permanyer. Este es un artículo open access bajo la licencia CC BY-NC-ND (http://creativecommons.org/licenses/by-nc-nd/4.0/). 


\section{Introducción}

La exacerbación de la crisis provocada por la pandemia de COVID-19 generó una importante demanda de modelos predictivos del riesgo posterior a la infección por SARS-CoV-2.' En el marco de este esfuerzo, se concretaron modelaciones predictivas encaminadas a apoyar procesos de planeación y gestión de recursos, afinar protocolos médicos y delinear políticas colectivas de prevención. ${ }^{1,2}$ No obstante, la modelación predictiva ha sido escasamente centrada en las personas con miras a generar un mayor involucramiento en la prevención individual consciente. Esta última desempeña un papel trascendente en la estructuración de una prevención colectiva, informada y, sobre todo, colaborativa. ${ }^{3}$

El objetivo de esta investigación fue explorar el universo de datos públicos disponibles sobre COVID-19 en México, ${ }^{4,5}$ con la finalidad de generar un modelo predictivo de la letalidad y su implementación en un cuestionario con preguntas accesibles a la población en general. La modelación fue elaborada con una función discriminante de casos positivos a COVID-19, a través del desenlace de curarse o fallecer, otorgando a cada una de las variables predictivas una puntuación por medio de la técnica de scoring. ${ }^{6,7}$ Para dar mayor difusión a esta herramienta, el cuestionario resultante puede convertirse en una aplicación disponible en línea en la que, además, se desplieguen consejos preventivos personalizados en función de la puntuación que se obtenga.

\section{Métodos}

Este estudio utilizó los datos abiertos sobre COVID19 acumulados al 26 de julio de 2020, publicados y actualizados diariamente por la Dirección General de Epidemiología de la Secretaría de Salud del gobierno federal. ${ }^{4}$ También se consideraron los datos abiertos del visualizador analítico para COVID-19 del Instituto Nacional de Estadística y Geografía. ${ }^{5}$

De la base de datos de la Secretaría de Salud ${ }^{4}$ fueron seleccionados aleatoriamente 8000 pacientes que permanecieron con vida más de dos meses después de ser positivos a SARS-CoV2 y 8000 que fueron positivos a la misma infección y que fallecieron. De la base de datos del Instituto Nacional de Estadística y Geografía ${ }^{5}$ se eligieron los municipios de residencia y sus variables a los cuales correspondían los 16000 pacientes analizados.
Tomando en cuenta que la mayoría de los datos analizados son expresados en categorías, se realizó la homogeneización a través de una segmentación en cuatro clases incrementales por medio del algoritmo de medias móviles. ${ }^{8}$ Los datos resultantes de $75 \%$ de los pacientes positivos sirvieron para calcular el modelo discriminante básico y los del $25 \%$ restante, para llevar a cabo el proceso de validación externa del modelo.

Del conjunto de variables exploradas por su cercanía temática y epistemológica con el estudio, resultaron seleccionadas 15 variables por su carácter discriminante significativo $\left(\chi^{2}\right.$ con $\left.p<0.05\right)$ de los desenlaces de curarse o fallecer ${ }^{9,10}$ (Tabla 1).

Enseguida, se realizó un análisis de correspondencias múltiples, conservando los 27 ejes $(73.67 \%$ de la varianza total explicada) que resultaron significativos $(p<0.05){ }^{6,11}$ Con estos factores se realizó el cálculo de la función lineal discriminante de Fisher, que permitió generar el modelo de predicción seguido por la atribución de coeficientes a las variables categoriales por medio de una función score ${ }^{7,12}$ (Tabla 2). La distribución de la puntuación definió la predicción de tres situaciones:

- El pronóstico óptimo de salvarse de la enfermedad.

- La predicción de alerta para pacientes proclives a fallecer.

- La indecisión, que agrupa a individuos positivos con una puntuación intermedia.

La evaluación del modelo desarrollado se realizó por medio de una validación interna con la técnica de bootstrap con 1000 remuestreos, ${ }^{13,14}$ la cual fue profundizada con el apoyo de la curva ROC (receiver operating characteristic) y la gráfica de LIFT. ${ }^{15}$ Se llevó a cabo también una validación externa con $25 \%$ de los individuos positivos, población que no participó en la generación del modelo.

\section{Resultados}

La evaluación de la calidad y eficiencia del modelo de predicción se llevó a cabo en dos pasos complementarios: la validación interna y la validación externa.

\section{La validación interna del modelo}

La validación de la estabilidad interna del modelo evidencia las variaciones mínimas entre el resultado del cálculo básico del modelo y los resultados con bootstrap. ${ }^{16}$ En todos los casos, esas diferencias 
Tabla 1. Características demográficas, clínicas y contextuales de la población analizada*

\begin{tabular}{|c|c|c|c|}
\hline Características & $\begin{array}{c}\text { Vivos } \\
(\%)\end{array}$ & $\begin{array}{c}\text { Fallecidos } \\
(\%)\end{array}$ & $\begin{array}{c}\text { Total } \\
(n=16000)\end{array}$ \\
\hline $\begin{array}{l}\text { V1. Sexo } \\
\text { Hombre } \\
\text { Mujer }\end{array}$ & $\begin{array}{l}26.11 \\
23.89\end{array}$ & $\begin{array}{l}32.41 \\
17.59\end{array}$ & $\begin{array}{l}9363 \\
6637\end{array}$ \\
\hline $\begin{array}{l}\text { V2. Grupos de edad } \\
1-4 \\
5-14 \\
15-24 \\
25-34 \\
35-44 \\
45-64 \\
\geq 65\end{array}$ & $\begin{array}{c}0.30 \\
0.57 \\
3.55 \\
11.61 \\
12.38 \\
17.13 \\
4.47\end{array}$ & $\begin{array}{l}0.08 \\
0.04 \\
0.24 \\
1.37 \\
4.18 \\
22.10 \\
22.00\end{array}$ & $\begin{array}{c}60 \\
98 \\
606 \\
2076 \\
2649 \\
6276 \\
4235\end{array}$ \\
\hline V3. Embarazo & 90.00 & 10.00 & $70(1.05)$ \\
\hline V4. Obesidad & 43.87 & 56.13 & 3476 (21.72) \\
\hline $\begin{array}{l}\text { Comorbilidad }{ }^{* *} \\
\text { V5. Diabetes } \\
\text { V6. Pulmonar obstructiva } \\
\text { crónica } \\
\text { V7. Inmunosupresión } \\
\text { V8. Hipertensión } \\
\text { V9. Cardiovascular } \\
\text { V10. Renal crónica } \\
\text { V11. Otras }\end{array}$ & $\begin{array}{l}24.90 \\
22.25 \\
\\
29.88 \\
26.37 \\
23.93 \\
15.73 \\
34.62 \\
\end{array}$ & $\begin{array}{l}75.10 \\
77.75 \\
70.12 \\
73.63 \\
76.07 \\
84.27 \\
65.38\end{array}$ & $\begin{array}{c}4061(25.38) \\
445(2.78) \\
\\
328(2.05) \\
4733(29.58) \\
560(3.5) \\
674(4.21) \\
621(3.88)\end{array}$ \\
\hline $\begin{array}{l}\text { V12. Pacientes positivos por } \\
\text { municipio } \\
1-948 \\
949-3228 \\
3229-6832 \\
6833-11587\end{array}$ & $\begin{array}{c}14.40 \\
15.19 \\
14.01 \\
6.40\end{array}$ & $\begin{array}{c}16.08 \\
13.61 \\
14.39 \\
5.91\end{array}$ & $\begin{array}{l}4876 \\
4608 \\
4543 \\
1970\end{array}$ \\
\hline $\begin{array}{l}\text { V13. Población de } 3 \text { a } 5 \text { años } \\
\text { que asiste a la escuela por } \\
\text { municipio } \\
\text { 0-49.36 } \\
\text { 49.37-61.76 } \\
61.77-74.69 \\
74.70-100\end{array}$ & $\begin{array}{c}2.68 \\
15.74 \\
23.20 \\
8.36\end{array}$ & $\begin{array}{c}3.44 \\
16.32 \\
23.16 \\
7.06\end{array}$ & $\begin{array}{c}979 \\
5129 \\
7417 \\
2466\end{array}$ \\
\hline $\begin{array}{l}\text { V14. Sector médico en donde } \\
\text { se atiende (población 15 894) } \\
\text { Estatal } \\
\text { IMSS } \\
\text { ISSSTE } \\
\text { Pemex } \\
\text { Municipal } \\
\text { Universitario } \\
\text { Privada } \\
\text { Sedena } \\
\text { Semar } \\
\text { Secretaría de Salud }\end{array}$ & $\begin{array}{c}1.13 \\
15.11 \\
1.86 \\
0.69 \\
0.04 \\
0.03 \\
1.77 \\
0.38 \\
0.52 \\
28.52\end{array}$ & $\begin{array}{c}1.15 \\
27.46 \\
3.72 \\
0.75 \\
0.04 \\
0.03 \\
0.58 \\
0.49 \\
0.23 \\
15.50\end{array}$ & $\begin{array}{c}362 \\
6765 \\
888 \\
229 \\
13 \\
9 \\
374 \\
138 \\
118 \\
6997\end{array}$ \\
\hline $\begin{array}{l}\text { V15. Nacido en otra entidad } \\
\text { que la de residencia }\end{array}$ & 10.99 & 13.06 & $3848(24.05)$ \\
\hline
\end{tabular}

fueron inferiores a $0.2 \%$, lo que indicó una buena estabilidad interna. En el mismo orden de ideas, la curva ROC destacó una puntuación límite de 575 , que caracteriza una sensibilidad de $76.6 \%$ y una especificidad de $23.1 \%$, ambas aceptables en el marco de los objetivos fijados a la presente investigación (Figura 1).

La capacidad discriminante del modelo por medio del área bajo la curva (AUC) fue de 0.845 , con un intervalo de confianza de $95 \%$ que indica que la predicción distingue entre curados y fallecidos con una probabilidad de $84.5 \%$. Un modelo con este valor de AUC es considerado útil para ciertos usos según los criterios de Swets, ${ }^{17}$ mientras que es caracterizado como excelente según los criterios de Hosmer y Lemeshow. $^{18}$

\section{Validación externa del modelo}

El comparativo de los resultados obtenidos con el modelo básico y el cálculo elaborado con $25 \%$ de los pacientes de prueba dejó entrever diferencias marginales (Tabla 3), las cuales quedaron contenidas en el rango inferior a $0.65 \%$ en valor absoluto, que no parece incidir sensiblemente en la eficiencia del modelo. Lo anterior puede verse en la gráfica LIFT, que revela un modelo capaz de determinar $76.57 \%$ de predicciones acertadas con $50 \%$ de los pacientes positivos (Figura 1).

El resultado de la función discriminante con variables categoriales fue utilizado para elaborar una función score; cada uno de los pacientes positivos fue asignado a tres posibles zonas a partir de la puntuación que obtuvieron. En nuestro caso, la puntuación elevada correspondió a la zona de alerta (622-1000) y la puntuación baja coincidió con la zona óptima (0-528). La zona de indecisión identificó a individuos con puntuación oscilante entre 528-622, quienes no pudieron clasificarse en ninguna de dos anteriores (Tabla 4).

\section{Discusión}

Por medio de la modelación discriminante se estableció la posibilidad de predicción de curación y fallecimiento al contraer el virus SARS-CoV-2 en México. El modelo de predicción generado tiene la ventaja de estar sustentado en datos públicos accesibles, consta de variables predictivas fáciles de contestar y, por lo tanto, susceptibles de facilitar la 
Tabla 2. Validación interna y externa de la función lineal discriminante de Fisher

\begin{tabular}{|c|c|c|c|c|}
\hline \multirow{2}{*}{$\begin{array}{l}\text { Elaboración del modelo } \\
\text { Grupos discriminados }\end{array}$} & \multicolumn{2}{|c|}{ Cálculo básico } & \multicolumn{2}{|c|}{ Cálculo con bootstrap* } \\
\hline & Bien clasificados & Mal clasificados & Bien clasificados & Mal clasificados \\
\hline $\begin{array}{l}\text { Positivos vivos } \\
\text { n } \\
\%\end{array}$ & $\begin{array}{l}4492 \\
74.85\end{array}$ & $\begin{array}{r}1508 \\
25.13\end{array}$ & $\begin{array}{c}4481.50 \\
74.69[0.63]\end{array}$ & $\begin{array}{c}1518.50 \\
25.31[0.63]\end{array}$ \\
\hline $\begin{array}{l}\text { Positivos fallecidos } \\
\text { n } \\
\%\end{array}$ & $\begin{array}{l}4761 \\
79.35\end{array}$ & $\begin{array}{r}1239 \\
20.65\end{array}$ & $\begin{array}{c}4751.61 \\
79.19[0.67]\end{array}$ & $\begin{array}{c}1248.39 \\
20.81[0.67]\end{array}$ \\
\hline $\begin{array}{c}\text { Total } \\
\text { n } \\
\%\end{array}$ & $\begin{array}{l}9253 \\
77.11\end{array}$ & $\begin{array}{l}2747 \\
22.89\end{array}$ & $\begin{array}{c}9233.11 \\
76.94[0.37]\end{array}$ & $\begin{array}{c}2766.89 \\
23.06[0.37]\end{array}$ \\
\hline Comprobación del modelo & \multicolumn{2}{|c|}{ Cálculo básico } & & \\
\hline $\begin{array}{l}\text { Positivos vivos } \\
\text { n } \\
\%\end{array}$ & $\begin{array}{r}1502 \\
75.10\end{array}$ & $\begin{array}{c}498 \\
24.90\end{array}$ & & \\
\hline $\begin{array}{l}\text { Positivos fallecidos } \\
\text { n } \\
\%\end{array}$ & $\begin{array}{c}1574 \\
78.70\end{array}$ & $\begin{array}{c}426 \\
21.30\end{array}$ & & \\
\hline $\begin{array}{c}\text { Total } \\
\mathrm{n} \\
\%\end{array}$ & $\begin{array}{l}3076 \\
76.90\end{array}$ & $\begin{array}{c}924 \\
23.10\end{array}$ & & \\
\hline
\end{tabular}

*1000 remuestreo aleatorio.

Entre corchete se indica la desviación estándar.

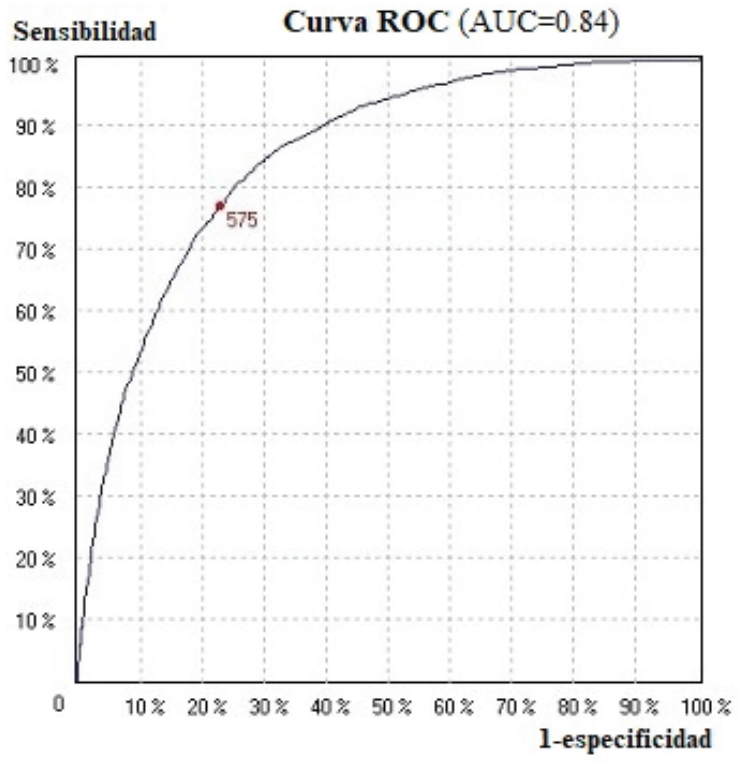

Figura 1. Calidad y eficiencia del modelo de predicción.

creación de una herramienta computacional con propósitos preventivos. Durante la crisis de COVID-19, Wynants et al. ${ }^{1}$ documentaron cerca de 145 modelos predictivos, $34 \%$ dedicados al pronóstico de riesgo de mortalidad o de necesidad de cuidados

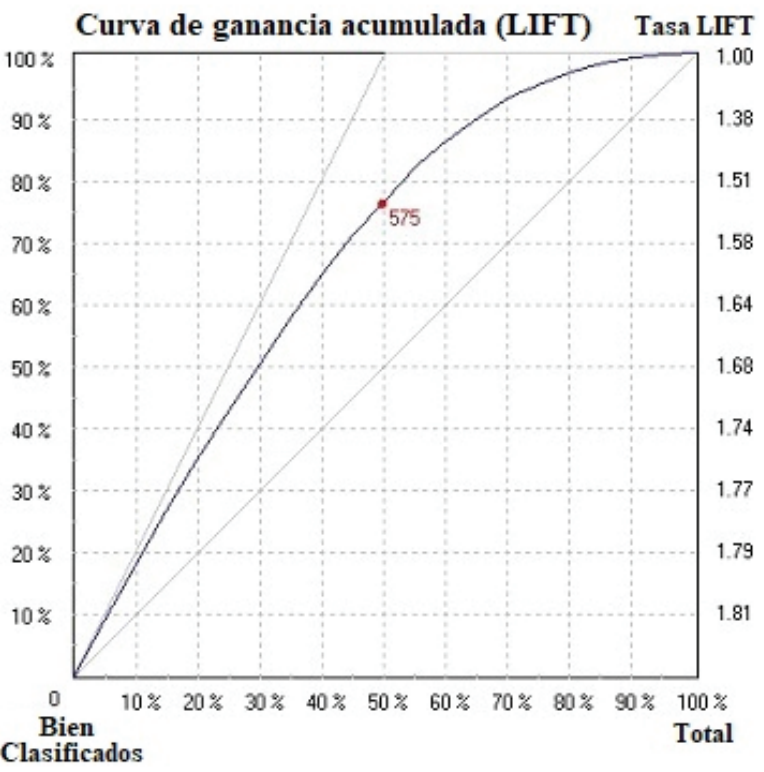

intensivos. En su mayoría, los modelos que mostraron avances en el conocimiento de la enfermedad ${ }^{2,19}$ fueron calificados de sesgados debido a la selección no representativa de pacientes control y al sobreajuste del modelo. ${ }^{1}$ 
Tabla 3. Coeficientes discriminantes y transformados de las modalidades involucradas

\begin{tabular}{|c|c|c|}
\hline Características & $\begin{array}{l}\text { Coeficientes } \\
\text { de la función } \\
\text { discriminante }\end{array}$ & $\begin{array}{l}\text { Coeficientes } \\
\text { transformados }\end{array}$ \\
\hline
\end{tabular}

V1. Sexo

Hombre

Mujer

V2. Grupos de edad (años)

1-4

5-14

$15-24$

25-34

$35-44$

$45-64$

$\geq 65$

\subsection{6}

$-2.731$

$-21.688$

$-28.67$

$-30.371$

$-29.564$

$-18.279$

4.641

24.365

15.85

0

V3. Embarazo

3.323

1.918

29.5

5.78

0

2.74

41.08

118.95

185.96

V4. Obesidad

Mennicipal

V5. Diabetes

V6. Pulmonar obstructiva crónica

V7. Inmunosupresión

V8. Hipertensión

v9. Cardiovascular

V10. Renal crónica

V11. Otras

Municipa

$-1.38$

3.735

3.981

1.918

13.754

2.487

V12. Pacientes positivos por

municipio

$1-948$

949-3228

3229-6832

6833-11587

3.67

$-1.735$

14.01

$-0.901$

20.47

46.73

V13. Población de 3 a 5 años que asiste a la escuela por municipio

$0-49.36$

49.37-61.76

$61.77-74.69$

$74.70-100$

6.038

0.297

$-0.327$

8.36

27.46

7.96

5.84

7.06

V14. Sector médico en donde

se atiende

Estatal $\quad 1.718$

$\begin{array}{lll}\text { ISSSTE } & 6.917 & 350.06 \\ \end{array}$

\begin{tabular}{lll} 
Municipal & -10.005 & 285.99 \\
\hline
\end{tabular}

Universitario $\quad-18.09 \quad 258.52$

\begin{tabular}{l|l|l|}
\hline Pemex & -8.596 & 290.77 \\
\hline
\end{tabular}

$\begin{array}{lll}\text { Privada } & -21.188 & 247.99\end{array}$

$\begin{array}{lll}\text { Sedena } & 5.287 & 337.94\end{array}$

$\begin{array}{lll}\text { Semar } & -9.127 & 288.97\end{array}$

$\begin{array}{lll}\text { Secretaría de Salud } & -8.069 & 292.56\end{array}$

V15. Nacido en otra entidad que la de residencia

\begin{tabular}{l|c|c} 
Sí & 1.065 & 4.76 \\
No & -0.337 & 0
\end{tabular}

IMSS = Instituto Mexicano del Seguro Social, ISSSTE $=$ Instituto de Seguridad y Servicios Sociales de los Trabajadores del Estado, Pemex = Petróleos Mexicanos, Sedena $=$ Secretaría de la Defensa Nacional, Semar $=$ Secretaría de Marina .
Tabla 4. Distribución de pacientes con una tasa tolerada de error de $10 \%$

\begin{tabular}{|l|c|c|c|c|c|c|}
\hline $\begin{array}{l}\text { Pacientes } \\
\text { positivos }\end{array}$ & \multicolumn{2}{|c|}{$\begin{array}{c}\text { Zona óptima } \\
(\mathbf{0 - 5 2 8} \text { puntos })\end{array}$} & \multicolumn{2}{|c|}{$\begin{array}{c}\text { Zona de } \\
\text { indecisión } \\
(\mathbf{5 2 8 - 6 2 2})\end{array}$} & $\begin{array}{r}\text { Zona de alerta } \\
\text { (622-1000 } \\
\text { puntos) }\end{array}$ \\
\cline { 2 - 7 } & $n$ & $\%$ & $n$ & $\%$ & $n$ & $\%$ \\
\hline Curados & 4777 & 59.71 & 2423 & 30.29 & 800 & 10 \\
\hline Fallecidos & 807 & 10.09 & 2992 & 37.40 & 4201 & 52.51 \\
\hline Total & 5584 & 34.90 & 5415 & 33.84 & 5001 & 31.26 \\
\hline
\end{tabular}

Aunque el presente estudio no reproduce las carencias metodológicas citadas, el modelo propuesto no parece ser el instrumento conveniente para la toma de decisiones de trascendencia operativa en el ámbito médico. Aún con un AUC de 0.845 , el modelo propuesto adquiriría más eficiencia con la incorporación de variables sintomáticas y de laboratorio. ${ }^{2}$ No obstante, lo anterior alejaría también el modelo de sus objetivos preventivos inducidos por la capacidad de una persona común de contestar instantáneamente un conjunto de preguntas. Tomando en cuenta la escasez de herramientas de pronóstico individual, ${ }^{1}$ el dilema en este estudio fue resuelto a favor de los alcances preventivos.

En términos teóricos, el modelo parece corroborar, como se identifica en otras investigaciones, la importancia de la edad, el sexo y la comorbilidad para el riesgo de letalidad. ${ }^{1,3}$ Como aportación específica de este estudio, se subraya el peso de factores contextuales como los sectores que atienden a los pacientes con COVID-19, el número de pacientes positivos y la tasa de niños de tres a cinco años que asisten a la escuela por municipio de residencia. La explicación de la incidencia de las dos primeras variables parece evidente, pero en el caso de la última, lo que más se acerca es un referente hipotético de una inmunidad social en esa categoría de infantes. ${ }^{20}$

En términos prácticos, el modelo desarrollado exhibe cualidades estadísticas que le permiten convertirse en una aplicación de carácter preventivo de 15 preguntas estructuradas a partir de las variables de la Tabla 3. En el marco de esta aplicación, se otorgará una puntuación (coeficiente transformado de la Tabla 3) a cada una de las modalidades de respuesta, misma que será sumada, una vez finalizado el cuestionario para la asignación del caso, a una de las tres zonas de decisión (de alerta, de indecisión y 
óptima). La asignación de la consulta a una zona de decisión podría alcanzar un nivel de utilidad acompañado de consejos médicos personalizados de carácter preventivo. ${ }^{21}$

\section{Conclusión}

Se obtuvo un modelo predictivo con un grado de utilidad que cuenta con un respaldo estadístico y nomológico que permite su conversión en una aplicación de carácter preventivo. Un ejemplo preliminar de esta aplicación puede consultarse en https://bit. Iy/2YPlox8

\section{Conflicto de intereses}

Los autores declaran no tener conflicto de interés alguno.

\section{Financiamiento}

Los autores no recibieron patrocinio para llevar a cabo este artículo.

\section{Responsabilidades éticas}

Protección de personas y animales. Los autores declaran que para esta investigación no se realizaron experimentos en seres humanos ni en animales.

Confidencialidad de los datos. Los autores declaran que en este artículo no aparecen datos de pacientes.

Derecho a la privacidad y consentimiento informado. Los autores declaran que en este artículo no aparecen datos de pacientes.

\section{Bibliografía}

1. Wynants L, van Calster B, Collins GS, Riley RD, Heinze G, Schuit E, et al. Prediction models for diagnosis and prognosis of COVID-19: systematic review and critical appraisal. BMJ. 2020;369:m1328.

2. Ciceria F, Castagnaa A, Rovere-Querinia P, de Cobellia F, Ruggerib A, Gallib L, et al. Early predictors of clinical outcomes of COVID-19 outbreak in Milan, Italy. Clin Immunol. 2020;217:108509.

3. Liu T, Liang W, Zhong H, He J, Chen Z, Guanhao H, et al. Risk factors associated with COVID-19 infection: a retrospective cohort study based on contacts tracing. Emerg Microbes Infec. 2020;9:1546-1553.

4. Dirección General de Epidemiología, Secretaría de Salud; [Internet]. México: Datos abiertos; 2020.

5. Instituto Nacional de Estadística y Geografía [Internet]. México: Visualizador analítico para el COVID-19; 2020.

6. Lebart L, Morineau A, Piron M. Statistique exploratoire multidimensionnelle. Francia: Dunod; 2000.

7. Steyerberg EW, Vickers AJ, Cook NR, Gerds T, Gonen M, Obuchowski N, et al. Assessing the performance of prediction models: a framework for traditional and novel measures. Epidemiology. 2010;21:128-138.

8. Bahmani B, Moseley B, Vattani A, Kumar R, Vassilvitskii S. Scalable k-means++. Proceedings of the VLDB Endowment. 2012;5:622-633.

9. Morineau A. Note sur la caractérisation statistiques d'une classe et les valeurs-tests. Bull Techn Centre Statist Inform Appl. 1984;2:20-27.

10. Nakacha JP, Confais J. Approche pragmatique de la classification. Francia: Technip; 2004.

11. Bardos M. Analyse discriminante. Application au risque et scoring financier. Francia: Dunod; 2001

12. Droesbeke JJ, Lejeune M, Saporta G, editores. Modèles statistiques explicative pour données qualitatives. Francia: Editions Technip; 2005

13. Bleeker SE, Moll HA, Steyerberg EW, Donders, G, Derksen-Lubsen DE, Grobbee SE, et al. External validation is necessary in prediction research: a clinical example. J Clin Epidemiol. 2003;56:826-832.

14. Wahl S, Boulesteix AL, Zierer A, Thorand B, van de Wiel MA. Assessment of predictive performance in incomplete data by combining internal validation and multiple imputation. BMC Med Res Methodol. 2016;16:144.

15. Vuk M, Curk, T. ROC curve, lift chart and calibration plot. Metodoloski zvezki. 2006;3:89-108

16. Xie J, Qu Z. Bootstrap technique for ROC analysis: a stable evaluation of Fisher classifier performance. J Electron. 2007:24:523-527.

17. Swets JA. Measuring the accuracy of diagnosis system. Science. 1988;240:1285-1293.

18. Hosmer DW, Lemeshow S. Applied logistic regression. EE. UU.: John Wiley and Sons; 2000.

19. Bello-Chavolla O, Bahena-López J, Antonio-Villa N, Vargas-Vázquez A, González-Díaz A, Márquez-Salinas C, et al. Predicting mortality due to SARS-CoV-2: a mechanistic score relating obesity and diabetes to COVID-19 outcomes in Mexico. J Clin Endocrinol Metab. 2020;105:1-10.

20. Kloc M, Ghobrial RM, Kuchard E, Lewickie S, Kubiak J. Development of child immunity in the context of COVID-19 pandemic. Clin Immunol. 2020;217:108510.

21. Barajas-Ochoa A, Andrade-Romo J, Ramos-Santillán V. Retos para la educación médica en México en los tiempos del COVID-19. Gac Med Mex. 2020;156:254-257. 